\title{
Modeling and Quantifying of the Global Wrong Way Risk
}

\section{Badreddine Slime}

Financial Risk Quant from ENSAE (Ecole Nationale de la Statistique et de l'Administration Economique), Paris, France

Email: badreddine.slime@gmail.com

How to cite this paper: Slime, B. (2017) Modeling and Quantifying of the Global Wrong Way Risk. Journal of Financial Risk Management, 6, 231-246.

https://doi.org10.4236/jfrm.2017.63017

Received: June 18, 2017

Accepted: August 8, 2017

Published: August 11, 2017

Copyright (C) 2017 by author and Scientific Research Publishing Inc. This work is licensed under the Creative Commons Attribution International License (CC BY 4.0).

http://creativecommons.org/licenses/by/4.0/

\begin{abstract}
The counterparty risk issue has become increasingly important in the world of finance. This risk is defined as the loss due to the counterparty default. The regulator uses the Credit Value Adjustment ( $C V A)$ to measure this risk. However, there is the independency assumption between the default and the exposure behind the $C V A$ computation and it is not verified on the financial market. This paper presents two mathematical models for the assessment and the quantification of the counterparty risk without this assumption. This kind of risk is known as Wrong Way Risk ( $W W R$ ). This study focuses on three approaches: empirical, copula and mixed model. The first one is based on the hazard rate modelling to express the correlation between the probability of default and the exposure. The second one is about calculating the WWR effect using copulas. The last one is a combination of both. There is another assumption that makes easier the CVA computation: The constant of the loss given default $(L G D)$. As we know this assumption is not verified because the $L G D$ could be deterministic or stochastic. Otherwise, it could lead to a correlation effect between the $L G D$, the exposure and the default, and we then obtain a Global Wrong Way Risk $(G W W R)$. Indeed, we propose a model allowing the $C V A$ quantification without these assumptions.
\end{abstract}

\section{Keywords}

Counterparty Risk, Credit Value Adjustment, Wrong Way Risk, Copulas

\section{Introduction}

The credit value adjustment ( $C V A)$ computation is based on the independency assumption between the exposure and the default. However, this assumption is not verified on the market, and we although have correlation between the probability of default $(P D)$ and the exposure at default $(E A D)$. Therefore, we get two 
types of this effect: The Wrong Way Risk ( $W W R$ ) when the correlation is positive and the Right Way Risk $(R W R)$ when the correlation in this case is negative. There is another effect appears when the $L G D$ becomes random and also depends on the default. Indeed, this could generate a correlation between the three variables that make the $C V A$ assessment. In this case, we could call this effect as the Global Wrong Way Risk ( $G W W R$ ). First, we will focus on the $W W R$ effect and we make the difference between two kinds:

- The systemic $W W R$ : this kind arises at the moment where the dependency between the exposure and the default is due to a macroeconomic factor. In this case, this factor increases the $E A D$ and the $P D$. If we take a put on the $C A C A 0$ index with some bank like Société Général $(S G)$ as the issuer, then the CAC40 spot impacts both of the exposure and the counterparty rating. In fact, this index is a systemic factor in the French market and the $S G$ is a part of the $C A C 40$ composition.

- The specific $W W R$ : on the other hand, this kind comes from a specific factor. For example, we get this effect when we have a put on the stock of the issuer as underlying.

There are several models allowing the CVA computation with the WWR component. We present some of these approaches:

- The Basel model ${ }^{1}$ (Basel Committee on Banking Supervision, 2010): this approach is the most straightforward one to add the correlation effect and it is used by the Basel committee to add the WWR effect in the counterparty credit risk ( $C C R$ ) charge. It is based on the multiplier coefficient $\alpha>1$, to explain this effect. The exposure at default is given by the formula bellow:

$$
E A D=\alpha \times E E P E
$$

With EEPE represents the expected effective positive exposure.

We have by default $\alpha=1.4$, whatever, banks could have values above 1.2. Other institutions use values bigger than the default one. The coefficient value is appreciated, on one hand, by the name concentration and that also means the portfolio granularity by counterparties. On the other hand, we have the correlation effects between assets of the same counterparty. The Basel II accords do not give details to manage the WWR. However, Basel III brings more precision to manage this kind of risk, focusing on three aspects:

$>$ Implementation of a detailed process to manage the $W W R$.

Advising banks to put more provision to cover the counterparty risk.

Explication of the approach to manage transactions containing the specific $W W R$.

The implementation of this model remains easy and could be automatically integrated to the existent model, but it has two drawbacks:

It does not give the contribution part of the $W W R$.

It consumes more capital requirement to cover the counterparty risk, because

${ }^{1}$ Basel Committee on Banking Supervision (2010), “Basel III: A Global Regulatory Framework for More Resilient Banks and Banking Systems”. 
the standard approach is designed for the worst case.

- The empirical approach: this approach uses the hazard rate according to the exposure. Indeed, the relation between these two quantities allows getting the diffusion of the $P D$, and then it could explain the correlation with the exposure. The $W W R$ modeling progresses into three steps:

The choice of the function that gives the relation between the hazard rate and the exposure. The diffusion calculation is performed for each time step:

$$
h(t)=p(V(t))
$$

With $V($.$) represents the exposure. Hull and White { }^{2}$ (Hull \& White 2012) suggests an exponential function to implement this method.

$>$ The PD computation is done using the below formula:

$$
P D(t)=1-\mathrm{e}^{-\int_{0}^{t} h(s) \mathrm{d} s}
$$

The computation of the CVA WWR using the Monte Carlo simulation. This step is deduced directly from the exposure diffusion.

This approach allows a straight integration within the existent $C V A$ model. In fact, it replaces the computed $P D$ s under the independency assumption with the new one using the dependency between the default and the exposure.

- The copula model: This approach is based on copulas to explain the relation between the default variable and the exposure. Rosen and Saunders ${ }^{3}$ (Rosen \& Saunders 2012) use the Vasicek model to make this dependency. They introduced the Gaussian copula to compute the expected exposure without the independency assumption. Their model is implemented in three steps:

$>$ The default is written as a latent variable and it is divided in two components. The first part represents the specific risk, and the second part is the systemic risk:

$$
Y=\rho Z+\sqrt{1-\rho^{2}} \epsilon
$$

where $Z$ and $\epsilon$ are two independent random variables and they follow a Gaussian distribution. The counterparty is deemed in default when $Y$ is lower than $\Phi^{-1}\left(P D_{t}\right)$. The conditional probability of default regarding to the systemic variable is expressed as:

$$
P D_{t}(Z)=\Phi\left(\frac{\Phi^{-1}\left(P D_{t}\right)-\rho Z_{t}}{\sqrt{1-\rho^{2}}}\right)
$$

With $P D_{t}$ represents the unconditional probability of default.

Future exposures are mapped to a market variable $X$ that also follows a Gaussian distribution. The relation between this variable and the exposure is:

$$
X=\Phi^{-1}(F(V))
$$

With $F$ represents the distribution function of exposure and it is uniform. ${ }^{2}$ J. Hull and A. White (2012), CVA and Wrong Way Risk, Financial Analysis Journal, 68, pp. 58-69. ${ }^{3}$ D. Rosen, and D. Saunders (2012), CVA Wrong Way Risk, Journal of Risk Management in Financial Institutions, 5(3) pp. 252-272. 
This model supposes that the two variables $X$ and $Z$ are linked to a bivariate joint distribution. This relation is expressed under a Gaussian copula with the correlation $\rho$ between both of variables. Bocker and Brunnbauer ${ }^{4}$ (Bocker \& Brunnbauer, 2014) generalized this concept to use others copulas. This approach allows a straight integration within the existent $C V A$ model.

The next section will be devoted to the $W W R$ mathematical modeling using a mixed model. Indeed, we will use the empirical model to build the diffusion of $P D$ s, and then we will explain the relation between the default and the exposure under copulas model.

\section{Mathematical Modeling of the WWR}

The Credit Value Adjustment $(C V A)$ is defined by the difference between the portfolio value without the counterparty default and with this component. The $C V A$ could be written as:

$$
C V A=V P^{\text {Risk Free }}-V P
$$

where $V P^{\text {Risk Free }}$ represents the risk free portfolio value, and $V P$ is the portfolio value taking in consideration the counterparty default.

Using this, we find the following formula:

$$
C V A=\mathbb{E}\left[L G D(\tau) \times \tilde{V}^{+}(\tau) \times P D(\tau)\right]
$$

With $\tau$ is the default time, $\tilde{V}^{+}(\tau)=\max (0, V(\tau) \times D(\tau)), D($.$) represents$ the discount factor, and $L G D$ defines the Loss Given Default that we deem constant in this section.

We also can write:

$$
C V A=L G D \int_{0}^{T} E E^{+}(\tau=t) \mathrm{d} F(t)
$$

where $E E^{+}(\tau=t)=\mathbb{E}\left[\tilde{V}^{+}(t) \mid \tau=t\right]$, and $F$ defines the distribution of the default.

First, we begin by modeling the probability of default using the empirical model. For this, we introduce the hazard rate concept that measures the counterparty default occurrence. Giving the assumption that the default frequency follows the Poisson density, we get the formula below:

$$
P D(t)=1-\mathbb{E}\left[\exp \left(-\int_{0}^{t} h(s) \mathrm{d} s\right)\right]
$$

The relation between the $h(t)$, and the exposure is written as:

$$
h(t)=p(t, \tilde{V}(t))
$$

With $p$ is defined positive since $h(t) \geq 0, \forall t \geq 0$.

This function should also verify the following relation under the assumption that the hazard rates curve is flat:

$$
\exp (-\bar{h}(t) \times t)=\exp \left(-\frac{s(t) \times t}{L G D}\right)
$$

${ }^{4}$ K. Bocker and M. Brunnbauer (2014), Path consistent Wrong Way Risk, Risk Magazine. 
With $\bar{h}$ defines the arithmetic average of simulated values until the date $t$, and $s(t)$ is the market spread with the maturity of $t$.

We deem the following function of the hazard rate:

$$
p\left(t, \tilde{V}_{t}\right)=\exp (a(t)+b(t) \times \tilde{V}(t))
$$

where $b(t)$ is a function of time and determines the correlation between $h$ and $\tilde{V}$, and $a(t)$ is a function of time.

We can find the stochastic derivative equation $(S D E)$ of the hazard rate by applying the Itô's lemma on the $p\left(t, \tilde{V}_{t}\right)$, and we get:

$$
\frac{\mathrm{d} h}{h}=\left(\frac{\partial a(t)}{\partial t}+\frac{1}{2} b^{2}(t) \times \sigma_{\tilde{V}}^{2}(t)\right) \mathrm{d} t+b(t) \times \sigma_{\tilde{V}}(t) \mathrm{d} W_{t}^{Q}
$$

With $\mathrm{d} \tilde{V}_{t}=\sigma_{\tilde{V}}(t) \mathrm{d} W_{t}^{Q}, \sigma_{\tilde{V}}(t)$ defines the volatility of $\tilde{V}_{t}$ and $W_{t}^{Q}$ represents the Brownian motion under the risk neutral measure.

We conclude that the hazard rate follows a log-normal distribution with parameters below:

$$
\mu_{h}(t)=\int_{0}^{t}\left(\frac{\partial a(s)}{\partial t}+\frac{1}{2} b^{2}(s) \times \sigma_{\tilde{V}}^{2}(s)\right) \mathrm{d} s, \sigma_{h}^{2}(t)=\int_{0}^{t} b^{2}(s) \times \sigma_{\tilde{V}}^{2}(s) \mathrm{d} s
$$

In our case, we more interest about the distribution of the time integration of the $h$. If we take the following approximation $\bar{h}(t)=\int_{0}^{t} h(s) \mathrm{d} s \approx \sum_{i=1}^{k} h\left(t_{i}\right) \Delta t$ and by using the Fenton-Wilkinson ${ }^{5}$ (Fenton, 1960) approach, this quantity then follows a log-normal distribution with parameters below:

$$
\mu_{\bar{h}}(t)=2 \ln \left(m_{1}\right)-\frac{1}{2} \ln \left(m_{2}\right), \sigma_{\bar{h}}^{2}(t)=\ln \left(m_{2}\right)-2 \ln \left(m_{1}\right)
$$

With,

$$
\begin{aligned}
& m_{1}=\mathbb{E}[\bar{h}(t)]=\sum_{i=1}^{k} \exp \left(\mu_{h}\left(t_{i}\right)+\frac{\sigma_{h}^{2}\left(t_{i}\right)}{2}\right), \\
& m_{2}=\mathbb{E}\left[\bar{h}(t)^{2}\right]=\sum_{i=1}^{k} \exp \left(2 \mu_{h}\left(t_{i}\right)+\sigma_{h}^{2}\left(t_{i}\right)\right)
\end{aligned}
$$

It also supposes that $h\left(t_{i}\right)$ are independent. We get the following result by applying the Laplace transform approximation:

$$
\begin{aligned}
& \mathbb{E}[\exp (-\bar{h}(t))]=\sum_{k=0}^{\infty} \frac{\mathbb{E}\left[\bar{h}^{k}(t)\right]}{k !}(-1)^{k}=\sum_{k=0}^{\infty} \frac{\left(-\exp \left(\mu_{\bar{h}}(t)+\frac{k \times \sigma_{\bar{h}}^{2}(t)}{2}\right)\right)^{k}}{k !} \\
& \text { If } \frac{k \times \sigma_{\bar{h}}^{2}(t)}{2} \approx 0, \text { then } \mathbb{E}[\exp (-\bar{h}(t))]=\exp \left(-\mathrm{e}^{\mu_{\bar{h}}(t)}\right)
\end{aligned}
$$

The calibration is made in each time of the Monte Carlo simulation. Indeed, we minimize the distance between the $P D$ model and the $P D$ market basing on spreads. So, we need to do this process $M$ times using the discretization form of the hazard rate:

${ }^{5}$ Fenton L. (1960) the sum of lognormal probability distribution in scatter transmission system, IEEE trans. Communication Systems, Vol 8, pp. 56-57. 


$$
h_{i j}=\exp \left(a_{i}+b_{i} \tilde{V}_{i j}+\sigma_{i} \varepsilon_{i j}\right)
$$

where $h_{i j}=h\left(t_{i}\right)$ and $\tilde{V}_{i j}=\tilde{V}\left(t_{i}\right)$ represent the $f^{\text {th }}$ simulation of the exposure and the hazard rate.

The Appropriate parameters are those who minimize the following quantity:

$$
\min _{a_{i}, b_{i}, \sigma_{i}}\left|\frac{1}{M} \sum_{j=1}^{M} \exp \left(-\sum_{i=1}^{k} h_{i j} \mathrm{~d} t\right)-\exp \left(-\frac{s_{k} \times t_{k}}{L G D}\right)\right|, 1 \leq k \leq N
$$

where $M$ is the Monte Carlo number of simulation, $N$ is the number of steps time, $s_{k}$ and represents the spread with maturity $t_{k}$.

Finally, when the parameters are computed, then the hazard rates are built sequentially at each time step. The next step allows to define the relation between the exposure and the probability of default distribution. Therefore, we use the copulas to get this relation, and we take the following definitions:

- The value of the discount exposure $\tilde{V}$ at time $s$ follows a distribution $G_{s}(\tilde{v})=\mathbb{P}(\tilde{V}(s) \leq \tilde{v}), \tilde{v} \in \mathbb{R}$ with $g$ her density function.

- The default time of the counterparty $\tau>0$ is a random variable and we note his distribution function by $F(t)=\mathbb{P}(\tau \leq t), t>0$ and with the density $f$.

- The joint distribution of $\tilde{V}$ and $\tau$ is defined by:

$$
\mathbb{P}(\tilde{V}(s) \leq \tilde{v}, \tau \leq t) \equiv J_{s}(\tilde{v}, t)=C_{s}\left(G_{s}(\tilde{v}), F(t)\right)
$$

where $C_{s}$ indicates the bivariate copula and we suppose $C_{s}$ is twice continuously differentiable function. The density function is written as:

$$
c_{s}(x, y)=\frac{\partial^{2} C_{s}(x, y)}{\partial x \partial y},(x, y) \in[0,1]^{2}
$$

The expected positive exposure (EPE) is equal to:

$$
E E^{+}(s)=\mathbb{E}\left[\tilde{V}^{+}(s) \mid \tau=s\right]=\int_{-\infty}^{+\infty} j(\tilde{v} \mid s) \times \tilde{v}^{+} \mathrm{d} \tilde{v}
$$

With $j(\tilde{v} \mid t)=\frac{j(\tilde{v}, t)}{f(t)}$

We also have:

$$
j(\tilde{v}, t)=c_{s}\left(G_{s}(\tilde{v}), F(t)\right) \times g_{s}(\tilde{v}) \times f(t) \Rightarrow j(\tilde{v} \mid t)=c_{s}\left(G_{s}(\tilde{v}), F(t)\right) \times g_{s}(\tilde{v})
$$

We then replace the value of the conditional density in the formula to get the following result:

$$
\begin{aligned}
E E^{+}(s) & =\int_{-\infty}^{+\infty} c_{s}\left(G_{s}(\tilde{v}), F(s)\right) \times \tilde{v}^{+} \times g_{s}(\tilde{v}) \mathrm{d} \tilde{v} \\
& =\mathbb{E}\left[c_{s}\left(G_{s}(\tilde{v}), F(s)\right) \times \tilde{V}^{+}(s)\right]
\end{aligned}
$$

By applying the strong law of large number, we obtain the following approximation of the EPE:

$$
\mathbb{E}\left[c_{s}\left(G_{s}(\tilde{v}), F(s)\right) \times \tilde{V}^{+}(s)\right] \approx \frac{1}{M} \sum_{j=1}^{M} c_{s}\left(G_{s}\left(\tilde{v}_{j}\right), F(s)\right) \times \tilde{V}_{j}^{+}(s)
$$

It remains one issue to complete those calculations; we are talking about the 
estimation of the correlation between the exposure and the probability of default. In fact, it is the requirement parameter to compute $c_{s}\left(G_{s}\left(\tilde{v}_{j}\right), F(s)\right)$. We suggest two approaches to do this estimation:

- The first one uses the $\operatorname{Spearman}^{6}$ (Daniel, 1990) correlation who is defined as:

$$
r\left(t_{i}\right)=1-\frac{6 \times \sum d_{k}}{K \times\left(K^{2}-1\right)}
$$

where $d_{k}=r g(P D)-r g(\tilde{V})$ presents the ranks difference between the exposure and the probability of default, and $K$ is the number of observations. We compute this correlation at each time step and we use the result of the hazard rate diffusion for this. Finally, we can estimate the correlation by $r=\frac{1}{N} \sum_{i=0}^{N} r\left(t_{i}\right)$.

- The second one is based on the minimization of the difference between the simulated survival probabilities at time $t_{i}$ using the hazard rate model and the copula at each time step:

$$
\min _{r\left(t_{i}\right)}\left|\frac{1}{M} \sum_{j=1}^{M}\left[\exp \left(-\sum_{i=1}^{k} h_{i j} \mathrm{~d} t\right)-c_{s}\left(G_{s}\left(\tilde{v}_{j}\right), F(s)\right)\right]\right|, 1 \leq k \leq N
$$

We then can estimate the correlation by $r=\frac{1}{N} \sum_{i=0}^{N} r\left(t_{i}\right)$.

We have now all components to complete the $C V A$ computation with the $W W R$ effect. It remains to calculate all $E E^{+}\left(t_{i}\right)$ and apply the trapezoid integration method regarding to the $P D$ s to get the value. The implementation of the mixed model approach will be done on the CAC40 European put option. We will use the HESTON ${ }^{7}$ (Heston, 1993) model to compute the put price that is based on the stochastic volatility. We then take the following assumptions:

- The Loss Given Default LGD is constant and equal to $60 \%$.

- The discretization of time space is done on 100 steps.

- The dividends are equals to 0 .

- The credit spread of the counterparty is constant and equal to $0.8 \%$.

- The $C A C 40$ put strike value is 4350 .

- The Credit Support Annex (CSA) contains a Margin Call with 10 days as Margin Dates and the calculation will be done with and without collateral.

The value of the correlation between exposures and defaults using Spearman is equal to $r=-0.101122$. The Figure 1 shows the evolution of the EPE regarding to the $P D$ with and without collateral using the Gaussian copula:

We conclude that the EPE increases with the $W W R$ effect and the $C V A$ will also have the same behavior. The Figure 2 displays this effect:

The WWR increases the CVA quantity with $30 \%$, and that proves its importance and impact on the counterparty risk measurement. The Table 1 summarizes these results.

${ }^{6}$ Daniel, Wayne W. (1990). "Spearman rank correlation coefficient". Applied Nonparametric Statistics (2nd ed.). Boston: PWS-Kent. pp. 358-365.

${ }^{7}$ Heston, S.L., (1993), A closed-form solution for options with stochastic volatility with applications to bond and currency options, Review of Financial Studies, Vol.6, No 2, pp. 327-343. 


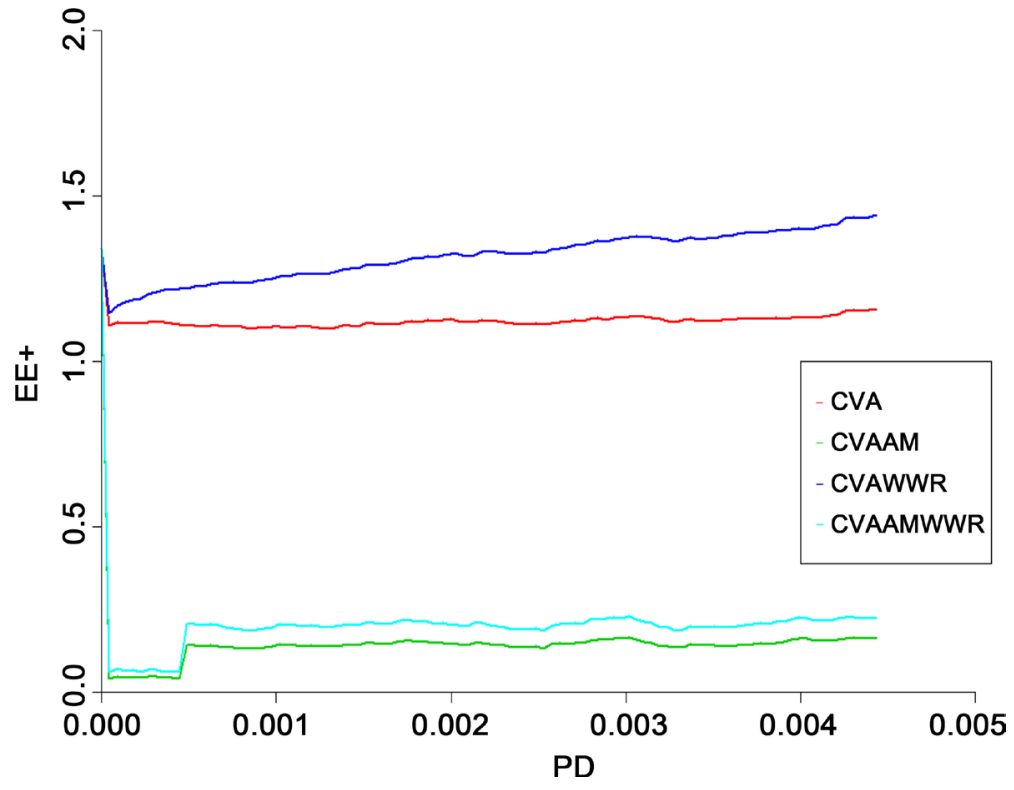

Figure 1. Expected positive exposure with and without $W W R$ effect.
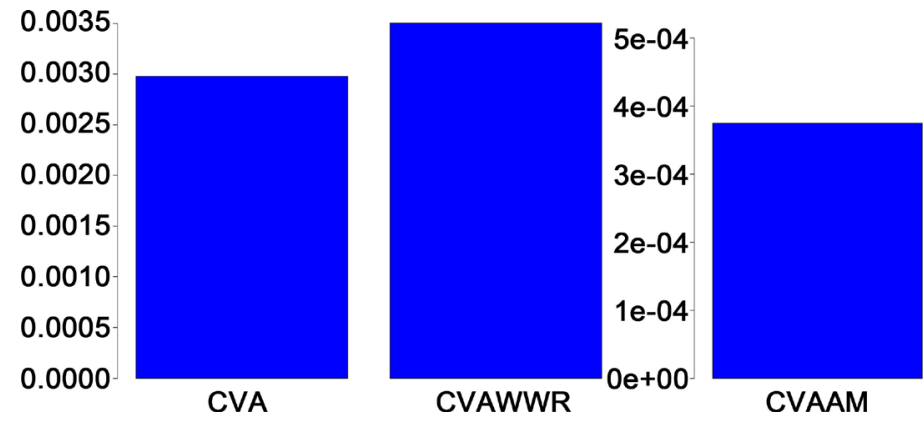

CVAAM

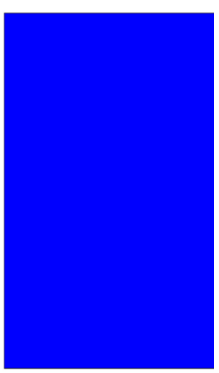

Figure 2. $C V A$ and $W W R$ effect with and without collateral.

Table 1. CVA WWR calculation results.

\begin{tabular}{ccc}
\hline & Without Collateral & With Collateral \\
\hline$C V A$ & 0.002979613 & 0.000374312 \\
$C V A(W W R)$ & 0.003501835 & 0.000521428
\end{tabular}

\section{The Global Wrong Way Risk (GWWR)}

As we saw in the last section, the compute of the $C V A$ supposes that the $L G D$ is constant. Furthermore, this assumption leads to the independency of this variable to the default. Nevertheless, the $L G D$ depends to the default and automatically to the exposure, because the $W W R$ proves that there is a dependency between the exposure and the default. We choose the word "Global" because we study the dependency between the three components that allow the calculation of the $C V A$. In order to model the Global Wrong Way Risk, we suggest two approaches:

- The first approach is based on the building of a relation between the $L G D$ and the exposure, and also, the definition of the relation between the exposure and the default. We use the same notation in the last section and we link 
the $L G D$ with the exposure through the below function:

$$
L G D(t)=L(\tilde{V}(t))
$$

With $\forall x \in R, L(x) \in[0,1]$.

We then replace in the $C V A$ formula:

$$
C V A^{G W W R}=\mathbb{E}\left[L G D(t) \times \tilde{V}^{+}(t) \times P D(t)\right]=\mathbb{E}\left[L(\tilde{V}(t)) \times \tilde{V}^{+}(t) \times P D(t)\right]
$$

There is two ways to compute this expectation, on one hand; we can make it with empirical approach. We define the relation below:

$$
h(t)=p(\tilde{V}(t))
$$

With $p$ is defined positive since $h(t) \geq 0, \forall t \geq 0$.

We so get the following result:

$$
\begin{aligned}
C V A^{G W W R} & =\int_{0}^{t} \mathbb{E}\left[L(\tilde{V}(s)) \times \tilde{V}^{+}(s) \times h(s) \times \exp \left(\int_{0}^{s} h(u) \mathrm{d} u\right)\right] \mathrm{d} s \\
& =\int_{0}^{t} \mathbb{E}\left[L(\tilde{V}(s)) \times \tilde{V}^{+}(s) \times p(\tilde{V}(s)) \times \exp \left(\int_{0}^{s} p(\tilde{V}(u)) \mathrm{d} u\right)\right] \mathrm{d} s
\end{aligned}
$$

By applying the strong law of large number, we have at each step of time $t_{k}, 1 \leq k \leq N$ the approximation of the Global expected positive exposure (GEPE):

$$
\begin{aligned}
G E E^{+}\left(t_{k}\right) & =\mathbb{E}\left[L(\tilde{V}(s)) \times \tilde{V}^{+}(s) \times p(\tilde{V}(s)) \times \exp \left(\int_{0}^{s} p(\tilde{V}(u)) \mathrm{d} u\right)\right] \\
& \approx \frac{1}{M} \sum_{j=1}^{M} L\left(\tilde{V}\left(t_{k}\right)\right) \times \tilde{V}^{+}\left(t_{k}\right) \times p\left(\tilde{V}\left(t_{k}\right)\right) \times \exp \left(\int_{0}^{t_{k}} p(\tilde{V}(u)) \mathrm{d} u\right)
\end{aligned}
$$

Using the trapezoid method for calculation the integral, we get:

$$
C V A^{G W W R} \approx \sum_{k=1}^{N}\left(\frac{G E E^{+}\left(t_{k}\right)+G E E^{+}\left(t_{k-1}\right)}{2}\right) \times\left(t_{k}-t_{k-1}\right)
$$

On the second hand, we can use the copula approach. Given the definitions in the last section, we have:

$$
E E^{+}(s)=\mathbb{E}\left[L(\tilde{V}(s)) \times \tilde{V}^{+}(s) \mid \tau=s\right]=\int_{-\infty}^{+\infty} j(\tilde{v} \mid s) \times \tilde{v}^{+} \times L(\tilde{v}) \mathrm{d} \tilde{v}
$$

Knowing that, $j(\tilde{v} \mid t)=c_{s}\left(G_{s}(\tilde{v}), F(t)\right) \times g_{s}(\tilde{v})$

We get the following result:

$$
\begin{aligned}
G E E^{+}(s) & =\int_{-\infty}^{+\infty} c_{s}\left(G_{s}(\tilde{v}), F(s)\right) \times \tilde{v}^{+} \times L(\tilde{v}) \times g_{s}(\tilde{v}) \mathrm{d} \tilde{v} \\
& =\mathbb{E}\left[c_{s}\left(G_{s}(\tilde{v}), F(s)\right) \times \tilde{V}^{+}(s) \times L(\tilde{V}(s))\right]
\end{aligned}
$$

By applying the strong law of large number, we obtain the following approximation of the GEPE:

$$
\begin{aligned}
& \mathbb{E}\left[c_{s}\left(G_{s}(\tilde{v}), F(s)\right) \times \tilde{V}^{+}(s) \times L(\tilde{V}(s))\right] \\
& \approx \frac{1}{M} \sum_{j=1}^{M} c_{s}\left(G_{s}\left(\tilde{V}_{j}\right), F(s)\right) \times \tilde{V}_{j}^{+}(s) \times L(\tilde{V}(s))
\end{aligned}
$$


We compute this quantity at each time step, and then we use the trapezoid method to compute the GWWR CVA.

The implementation of this approach needs to choose the link function between the $L G D$ and the exposure. We then suggest the following function:

$$
L(\tilde{V}(t))=1-b \times \mathrm{e}^{-a \times P D(t)}
$$

where $P D(t)=1-\mathbb{E}\left[\exp \left(-\int_{0}^{t} p(\tilde{V}(s)) \mathrm{d} s\right)\right.$

The calibration of the $L G D$ model could be done by defining the maximum and the minimum of the $L G D$. If we note respectively $L G D_{\min }$ and $L G D_{\max }$ the lower and the upper bound, we thus obtain:

$$
\left\{\begin{array}{l}
a=-\ln \left(\frac{1-L G D_{\text {max }}}{1-L G D_{\text {min }}}\right) \\
b=1-L G D_{\text {min }}
\end{array}\right.
$$

In our case, we take $L G D_{\min }=0.6$ and $L G D_{\max }=0.99$. We get the following values of $a=19.81, b=0.4$

The Figure 3 displays the evolution of the GEPE regarding to the $P D$ with and without collateral using the Gaussian copula:

We deduce that the GEPE increases with the $G W W R$ effect and the $C V A$ will also have the same behavior. The GWWR grows the CVA quantity with $100 \%$, and that proves its importance and impact on the counterparty risk measurement. The Table 2 summarizes these results:

The Figure 4 shows this effect:

- The second approach is built around the approximation of the difference between the classical $C V A$, and the other one without any assumptions using a close formula. We suppose that the default is driven via a systemic factor

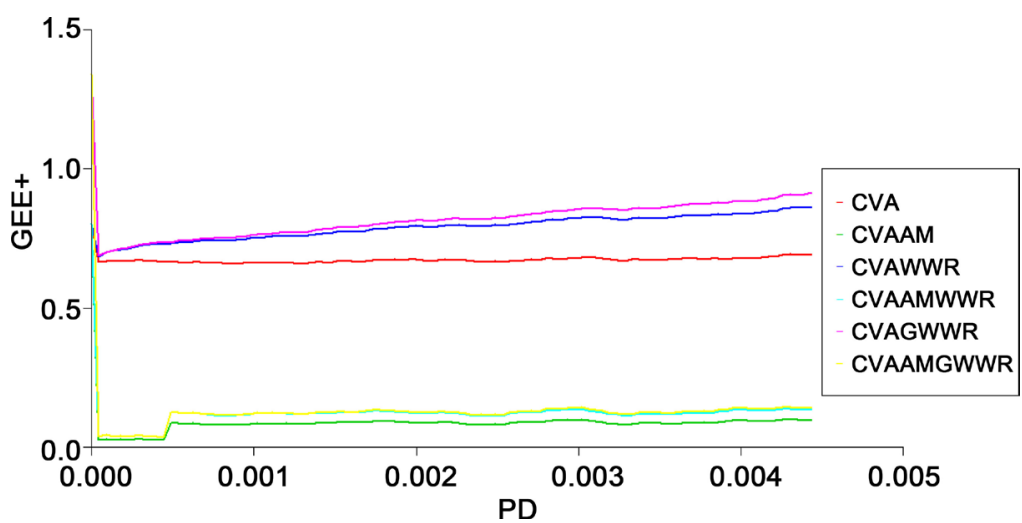

Figure 3. Expected positive exposure with and without $W W R$ effect.

Table 2. CVA GWWR calculation results.

\begin{tabular}{ccc}
\hline & Without Collateral & With Collateral \\
\hline$C V A$ & 0.002979613 & 0.000374312 \\
$C V A(W W R)$ & 0.003535026 & 0.000540142 \\
$C V A(G W W R)$ & 0.005891711 & 0.000900237 \\
\hline
\end{tabular}




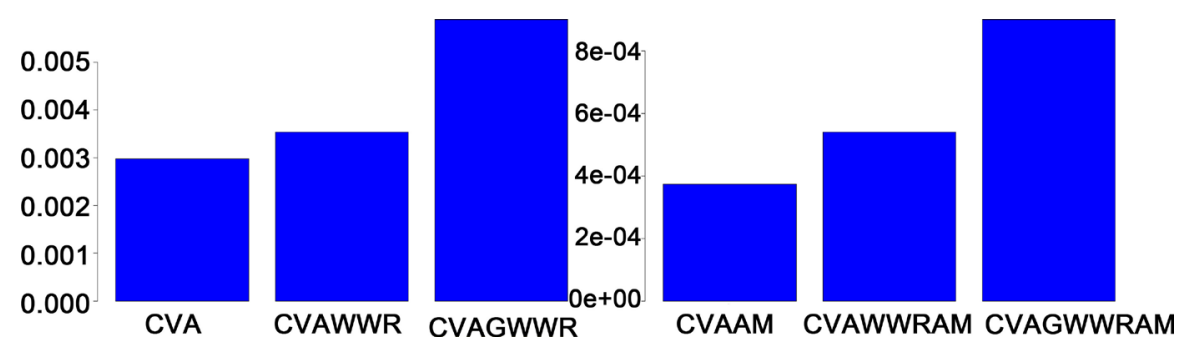

Figure 4. $C V A$ and $G W W R$ effect with and without collateral.

$X$ and the default arises at time $t$ when the $X$ reaches some level $x$ :

$$
\{\tau=t\} \equiv\{X=x\}
$$

We can write the $C V A$ under to the independency assumption as:

$$
\begin{aligned}
C V A^{I} & =\mathbb{E}[L \mid X]=\mathbb{E}\left[L G D(\tau) \times \tilde{V}^{+}(\tau) \times \mathbb{1}_{\{\tau \leq T\}} \mid X\right] \\
& \approx \sum_{k=1}^{N} \mathbb{E}\left[L G D\left(t_{k}\right)\right] \times \mathbb{E}\left[\bar{V}^{+}\left(t_{k}\right)\right] \times\left(F\left(t_{k} \mid X\right)-F\left(t_{k-1} \mid X\right)\right)
\end{aligned}
$$

With $\bar{V}^{+}\left(t_{k}\right)=\frac{\tilde{V}^{+}\left(t_{k}\right)+\tilde{V}^{+}\left(t_{k-1}\right)}{2}$

We note the Global Wrong Way Risk $C V A$ by $C V A^{G W W R}$ and we define the following function:

$$
D(\varepsilon)=C V A^{I}+\varepsilon \times\left(C V A^{G W W R}-C V A^{I}\right), \forall \varepsilon \in[0,1]
$$

For $\varepsilon=1$, we have $C V A^{G W W R}=C V A^{I}+\varepsilon \times\left(C V A^{G W W R}-C V A^{I}\right)$.

We define the following quantities:

$$
\begin{gathered}
\mu(X)=\sum_{k=1}^{N} \mu_{t_{k}}(X), \mu_{t_{k}}(X)=\mathbb{E}\left[L G D\left(t_{k}\right)\right] \times \mathbb{E}\left[\bar{V}^{+}\left(t_{k}\right)\right] \times\left(F\left(t_{k} \mid X\right)-F\left(t_{k-1} \mid X\right)\right) \\
\sigma^{2}(X)=\sum_{k=1}^{N} \sigma_{t_{k}}^{2}(X), \sigma_{t_{k}}^{2}(X)=\mathbb{V}\left[L G D\left(t_{k}\right) \times \bar{V}^{+}\left(t_{k}\right) \times \mathbb{1}_{\left\{t_{k-1} \leq \tau \leq t_{k}\right\}} \mid X\right]
\end{gathered}
$$

Under the assumption of independency of $L G D\left(t_{k}\right) \times \bar{V}^{+}\left(t_{k}\right) \times_{\left\{t_{k-1} \leq \tau \leq t_{k}\right\}} \mid X$, $\forall k=1, \cdots, N$. Using these notations, the Global Wrong Way Adjustment $(G W W A)$ is defined as:

$$
G W W A_{q}(C V A)=\operatorname{VaR}_{q}\left(C V A^{G W W R}\right)-\operatorname{VaR}_{q}\left(C V A^{I}\right)
$$

For a $q \in[0,1]$.

By applying the Taylor expansion on $\operatorname{VaR}_{q}(D(\varepsilon))$ with second order according to the $\varepsilon=0$ and by replacing the $\varepsilon=1$, we get:

$$
G W W A_{q}(C V A) \approx \frac{\partial}{\partial \varepsilon} \operatorname{VaR}_{q}(\mu(X))+\frac{1}{2} \frac{\partial^{2}}{\partial \varepsilon^{2}} \operatorname{VaR}_{q}(\mu(X))
$$

By computing the first and the second derivative terms, we find the following results $^{8}$ (Gourieroux, Laurent, \& Scaillet, 2000):

$$
\frac{\partial}{\partial \varepsilon} \operatorname{VaR}_{q}(\mu(X))=\mathbb{E}\left[L-\mu(X) \mid \mu(X)=\operatorname{VaR}_{q}(X)\right]=0
$$

${ }^{8}$ C. Gourieroux, J.P. Laurent, O. Scaillet (2000), Sensitivity analysis of Values at Risk, Journal of Empirical Finance. 


$$
\frac{\partial^{2}}{\partial \varepsilon^{2}} \operatorname{VaR}_{q}(\mu(X))=-\left.\left[\frac{1}{2 f_{X}(x)} \times \frac{\partial}{\partial x}\left(\frac{\sigma^{2}(x) f_{X}(x)}{\mu^{\prime}(x)}\right)\right]\right|_{x=V a R_{1-q}(X)}
$$

where $f_{X}$ represents the density function of the systemic factor.

We then find the result bellow ${ }^{9}$ (Slime, 2016):

$$
G W W A_{q}(C V A)=-\left.\frac{1}{2 \mu^{\prime}(x)}\left[\sigma^{2}(x) \times\left(\frac{f_{X}^{\prime}(x)}{f_{X}(x)}-\frac{\mu^{\prime \prime}(x)}{\mu^{\prime}(x)}\right)+\sigma^{2}(x)^{\prime}\right]\right|_{x=V a R_{1-q}(X)}
$$

We need to define a model for computing this quantity, and we then chose the CreditRisk $+{ }^{10}$ (Credit Suisse Financial Products, 1997) model. This model supposes that $X \sim \Gamma(\alpha, \beta)$ where $\alpha \times \beta=1$ and we obtain the following relation:

$$
\begin{gathered}
f_{X}(x)=\frac{1}{\beta^{\alpha} \Gamma(\alpha)} \mathrm{e}^{-\alpha x} x^{\alpha-1}, \frac{f_{X}^{\prime}(x)}{f_{X}(x)}=\frac{(\alpha-1)}{x}-\alpha \\
F\left(t_{k} \mid X=x\right)=F\left(t_{k}\right) \times(1-w+w \times x)
\end{gathered}
$$

Which $w$ represents the dependence factor between the counterparty and the systemic factor.

We also need to develop the derivative terms to complete the calculation, so we have:

$$
\begin{aligned}
\mu_{t_{k}}(x) & =\mathbb{E}\left[L G D\left(t_{k}\right)\right] \times \mathbb{E}\left[\bar{V}^{+}\left(t_{k}\right)\right] \times\left(F\left(t_{k} \mid X=x\right)-F\left(t_{k-1} \mid X=x\right)\right) \\
& =\mathbb{E}\left[L G D\left(t_{k}\right)\right] \times \mathbb{E}\left[\bar{V}^{+}\left(t_{k}\right)\right] \times\left(F\left(t_{k}\right)-F\left(t_{k-1}\right)\right) \times(1-w+w \times x)
\end{aligned}
$$

The $C V A$ assumption is the independency between the $L G D$, the exposure and the default, and this allows us to compute the following term:

$$
\begin{gathered}
\sigma_{t_{k}}^{2}(x)=\mathbb{V}\left[L G D\left(t_{k}\right) \times \bar{V}^{+}\left(t_{k}\right) \times \mathbb{1}_{\left\{t_{k-1} \leq \tau \leq t_{k}\right\}} \mid x\right] \\
=\mathbb{E}\left[\left(L G D\left(t_{k}\right) \times \bar{V}^{+}\left(t_{k}\right) \times \mathbb{1}_{\left\{t_{k-1} \leq \tau \leq t_{k}\right\}}\right)^{2} \mid x\right]-\left(\mathbb{E}\left[L G D\left(t_{k}\right) \times \bar{V}^{+}\left(t_{k}\right) \times \mathbb{1}_{\left\{t_{k-1} \leq \tau \leq t_{k}\right\}} \mid x\right]\right)^{2} \\
\mathbb{E}\left[\left(L G D\left(t_{k}\right) \times \bar{V}^{+}\left(t_{k}\right) \times \mathbb{1}_{\left\{t_{k-1} \leq \tau \leq t_{k}\right\}}\right)^{2} \mid x\right] \\
=\mathbb{E}\left[\left(L G D\left(t_{k}\right)\right)^{2}\right] \times \mathbb{E}\left[\left(\bar{V}^{+}\left(t_{k}\right)\right)^{2}\right] \times \mathbb{E}\left[\left(\mathbb{1}_{\left\{t_{k-1} \leq \tau \leq t_{k}\right\}}\right)^{2} \mid x\right]
\end{gathered}
$$

The second assumption of the CreditRisk+ model is that $\mathbb{1}_{\left\{t_{k-1} \leq \tau \leq t_{k}\right\}} \mid X$ follows a Poisson distribution with $\left(F\left(t_{k} \mid X=x\right)-F\left(t_{k-1} \mid X=x\right)\right)$ as intensity. So, we deduce that:

$\mathbb{E}\left[\left(\mathbb{1}_{\left\{t_{k-1} \leq \tau \leq t_{k}\right\}}\right)^{2} \mid x\right]=F\left(t_{k} \mid X=x\right)-F\left(t_{k-1} \mid X=x\right)+\left(F\left(t_{k} \mid X=x\right)-F\left(t_{k-1} \mid X=x\right)\right)^{2}$

${ }^{9}$ Slime, B. (2016). Credit Name Concentration Risk: Granularity Adjustment Approximation. Journal of Financial Risk Management, 5, 246-263.

${ }^{10}$ Credit Suisse Financial Products (1997). Credit Risk+: A Credit Risk Management Framework. London, 1997. 
We get the result bellow:

$$
\begin{gathered}
\sigma_{t_{k}}^{2}(x)=C_{t_{k}} \times \mu_{t_{k}}(x)+D_{t_{k}} \times\left(\mu_{t_{k}}(x)\right)^{2} \\
\text { With } C_{t_{k}}=\frac{\left(\mathbb{V}\left[L G D\left(t_{k}\right)\right]+\mathbb{E}\left[L G D\left(t_{k}\right)\right]^{2}\right)\left(\mathbb{V}\left[\bar{V}^{+}\left(t_{k}\right)\right]+\mathbb{E}\left[\bar{V}^{+}\left(t_{k}\right)\right]^{2}\right)}{\mathbb{E}\left[L G D\left(t_{k}\right)\right] \mathbb{E}\left[\bar{V}^{+}\left(t_{k}\right)\right]} \\
\text { And } D_{t_{k}}=\left(\frac{\mathbb{V}\left[L G D\left(t_{k}\right)\right]}{\mathbb{E}\left[L G D\left(t_{k}\right)\right]^{2}}+\frac{\mathbb{V}\left[\bar{V}^{+}\left(t_{k}\right)\right]}{\mathbb{E}\left[\bar{V}^{+}\left(t_{k}\right)\right]^{2}}+\frac{\mathbb{V}\left[L G D\left(t_{k}\right)\right] \mathbb{V}\left[\bar{V}^{+}\left(t_{k}\right)\right]}{\mathbb{E}\left[L G D\left(t_{k}\right)\right]^{2} \mathbb{E}\left[\bar{V}^{+}\left(t_{k}\right)\right]^{2}}\right)
\end{gathered}
$$

We conclude that:

$$
\begin{gathered}
\sigma^{2}(x)=\sum_{k=1}^{N}\left(C_{t_{k}}+D_{t_{k}} \times \mu_{t_{k}}(x)\right) \times \mu_{t_{k}}(x) \\
\sigma^{2}(x)^{\prime}=\sum_{k=1}^{N}\left(C_{t_{k}}+2 \times D_{t_{k}} \times \mu_{t_{k}}(x)\right) \times \mu_{t_{k}}^{\prime}(x)
\end{gathered}
$$

Subtitling in the GWWA formula, we obtain:

$$
G W W A_{q}(C V A)=-\left.\frac{1}{2 \mu^{\prime}(x)}\left[\left(\frac{(\alpha-1)}{x}-\alpha\right) \times \sigma^{2}(x)+\sigma^{2}(x)^{\prime}\right]\right|_{x=V a R_{1-q}(x)}
$$

If we note the classical $C V A$ by:

$$
C V A_{C}^{I}=\sum_{k=1}^{N} C V A_{C}^{I}\left(t_{k}\right)=\sum_{k=1}^{N} \mathbb{E}\left[L G D\left(t_{k}\right)\right] \times \mathbb{E}\left[\bar{V}^{+}\left(t_{k}\right)\right] \times\left(F\left(t_{k}\right)-F\left(t_{k-1}\right)\right)
$$

Then we have: $\mu^{\prime}(x)=w \times C V A_{C}^{I}$ and $\mu_{t_{k}}(x)=(1-w+w \times x) \times C V A_{C}^{I}\left(t_{k}\right)$ Finally, we get the following formula:

$G W W A_{q}(C V A)=\frac{1}{2 \times C V A_{C}^{I}} \sum_{k=1}^{N} C_{t_{k}} \times C V A_{C}^{I}\left(t_{k}\right) \times\left[\left(\vartheta-\frac{D_{t_{k}}}{C_{t_{k}}} \times C V A_{C}^{I}\left(t_{k}\right) \times(\vartheta+1)\right)\right]$

With $\theta(q, X)=\frac{\left(1-w+w \times \operatorname{VaR}_{1-q}(X)\right)}{w}$

And $\vartheta=\left(\left(\frac{(1-\alpha)}{V_{a R_{1-q}}(X)}+\alpha\right) \times \theta(q, X)-1\right)$

Under the assumption of $\left(F\left(t_{k}\right)-F\left(t_{k-1}\right)\right)^{2} \approx 0 \Rightarrow C V A_{C}^{I}\left(t_{k}\right)^{2} \approx 0$, we obtain the simplified formula of $G W W A$ :

$$
G W W A_{q}^{S}(C V A)=\frac{\vartheta}{2 \times C V A_{C}^{I}} \sum_{k=1}^{N} C_{t_{k}} \times C V A_{C}^{I}\left(t_{k}\right)
$$

Furthermore, the Global Wrong Way Risk CVA may be approximate using the formula bellow in the case of a symmetric distribution:

$$
C V A^{G W W R} \approx C V A^{I}+G W W A_{q}(C V A), q=0.5
$$

For non-symmetric distribution, we have:

$$
C V A^{G W W R} \approx C V A^{I}+\int_{0}^{1} G W W A_{q}(C V A) \mathrm{d} q
$$

As we know that the systemic factor $X$ follows the Gamma distribution, we 
could calibrate the factor $\alpha$ using the Maximum Likelihood Estimation $(M L E)$. We deem $n$ observation of $X=\left(X_{1}, \cdots, X_{n}\right)$ and the likelihood function is defined by:

$$
l(x, \alpha)=\prod_{i=1}^{n} \frac{\alpha^{\alpha}}{\Gamma(\alpha)} \mathrm{e}^{-\alpha x_{i}} x_{i}^{\alpha-1}=\left(\frac{\alpha^{\alpha}}{\Gamma(\alpha)}\right)^{n} \times \mathrm{e}^{-\alpha \sum_{i=1}^{n} x_{i}} \times \prod_{i=1}^{n} x_{i}^{\alpha-1}
$$

We then should compute the maximum of the logarithmic function:

$$
L(x, \alpha)=n \times(\alpha \times \ln (\alpha)-\ln (\Gamma(\alpha)))+\alpha \times\left(\sum_{i=1}^{n}\left(\ln \left(x_{i}\right)-x_{i}\right)\right)-\sum_{i=1}^{n} \ln \left(x_{i}\right)
$$

It remains to develop the first and the second derivative. The calculation leads to the following results:

$$
\frac{\partial L(x, \alpha)}{\partial \alpha}=0 \Rightarrow \ln (\hat{\alpha})+1-\frac{\Gamma^{\prime}(\hat{\alpha})}{\Gamma(\hat{\alpha})}=\frac{1}{n} \times\left(\sum_{i=1}^{n}\left(x_{i}-\ln \left(x_{i}\right)\right)\right)
$$

We use the Stirling approximation to resolve this equation:

$$
\ln (\Gamma(\alpha)) \approx\left(\alpha-\frac{1}{2}\right) \times \ln (\alpha)-\alpha-\ln (\sqrt{2 \pi}) \Rightarrow \hat{\alpha}=\frac{n}{2} \times\left(\sum_{i=1}^{n}\left(x_{i}-\ln \left(x_{i}\right)\right)-1\right)^{-1}
$$

We compute the estimator, we then get $\hat{\alpha}=0.324$. This estimator must verify the second condition, and we obtain by computing the second derivative:

$$
\frac{\partial^{2} L(x, \alpha)}{\partial \alpha^{2}}=-\frac{n}{2 \hat{\alpha}^{2}}<0
$$

The Figure 5 and the Table 3 summarize the comparison between all approaches. The approximation of the GWWR using the adjustment has a tow strong advantage. The first one, it gives us a closed formula to compute the $G W W R$ part. The second one, his implementation is straightforward and it

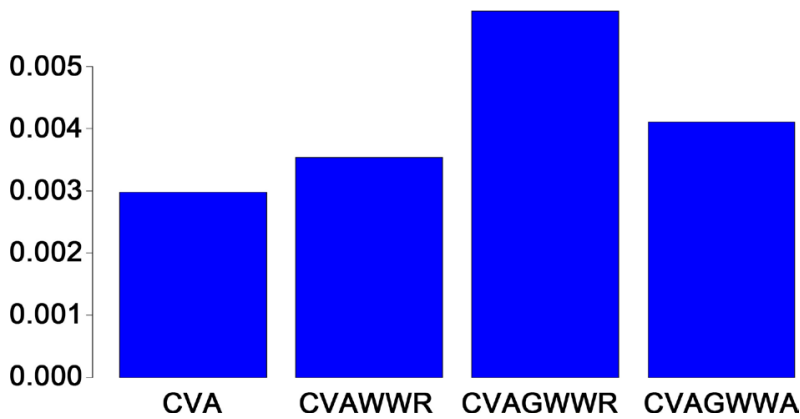

Figure 5. $C V A$ and $G W W A$ effect without collateral.

Table 3. $C V A G W W A$ calculation results.

\begin{tabular}{cc}
\hline & Without Collateral \\
\hline$C V A$ & 0.002979613 \\
$C V A(G W W R)$ & 0.003535026 \\
$C V A(G W W A)$ & 0.005891711 \\
\hline
\end{tabular}




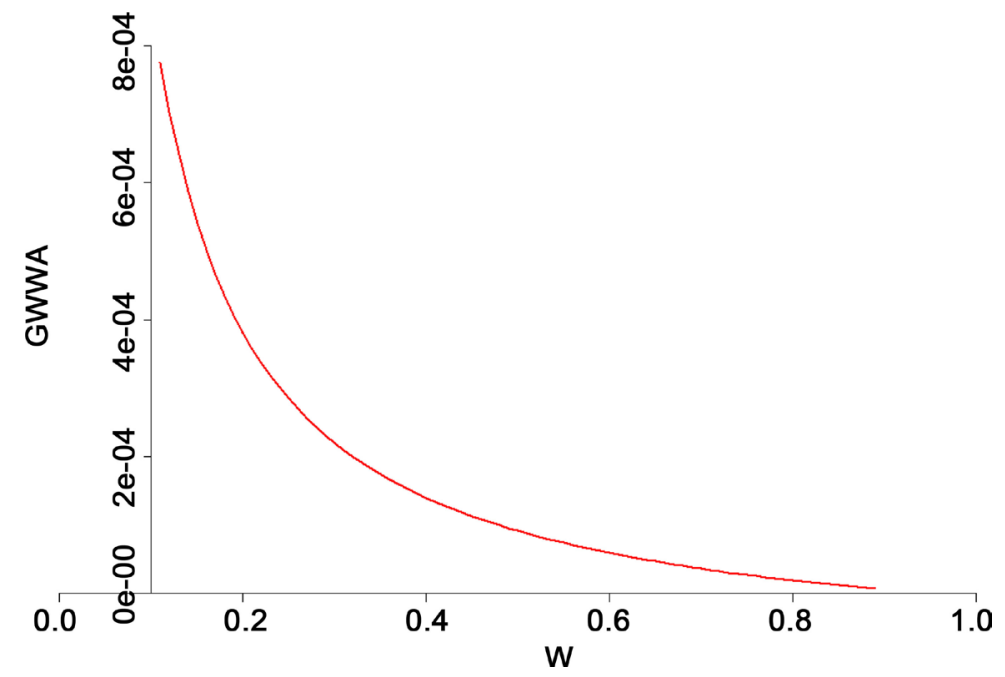

Figure 6. The evolution of $G W W A$ regarding to the correlation.

could be directly integrated on the existent framework.

We also conclude in the Figure 6 that the conditional default probability decreases within w, and the GWWA follows the same effect. This makes sense with the definition of the $G W W R$. Indeed, the exposure should also decrease regarding to the conditional $P D$, and the $G W W A$ must be reduced.

\section{Conclusion}

This paper was dedicated to the models allowing, on one hand, the quantifying of the Wrong Way Risk. On the other hand, we also developed two methods to integrate the Loss Given Default correlation effect. First, we began by introducing the existent approaches that give us the measurement of the WWR effect when we observe the positive correlation between the exposure and the default. We proposed a new model that combines the empirical and the copulas model. We made implementation on the European $C A C 40$ put, and we conclude that the $C V A$ increases potentially with the $W W R$ effect.

Then, we generalized the concept to deem the correlation effects between the three variables. So we added the $L G D$ correlation effect, and we proposed two models in this way. The first one is based on the definition of the function that links the $L G D$ with the default and we also used the copula to compute the conditional expectation exposure. The second one defines a close formula to compute the difference between the classical $C V A$ and the other one without the independency assumption.

We implemented both of these models on the European $C A C 40$ put, and we concluded that the $G W W R$ is more important than the $W W R$ in term of the $C V A$ level. Furthermore, the $G W W A$ allows a direct integration and computation of the $G W W R$ and we can also apply this model to the $W W R$. However, both of models represent some weakness. The first one needs to define and calibrate the $L G D$ model, and the integration of the existent model is not straightforward and will cost more time calculation. The second one remains an 
approximation of the $G W W R$ and requests a calibration of the systemic factor. We tried to give a close formula to allow a direct integration on the existent $C V A$ system, because the implementation arises one of most issues in the banking platform. By the way, we suggest getting more researching on the approaches that allow a straight integration.

\section{References}

Basel Committee on Banking Supervision (2010). Basel III: A Global Regulatory Framework for More Resilient Banks and Banking Systems. http://www.bis.org/publ/bcbs189_dec2010.pdf, December.

Bocker, K., \& Brunnbauer, M. (2014). Path Consistent Wrong Way Risk. Risk Magazine, 27, 49-53.

Credit Suisse Financial Products (1997). Credit Riskt: A Credit Risk Management Framework. London: Credit Suisse Financial Products.

Daniel, W. W. (1990). Spearman Rank Correlation Coefficient. Applied Nonparametric Statistics (2nd ed., pp. 358-365). Boston, MA: PWS-Kent.

Fenton, L. (1960). The Sum of Lognormal Probability Distribution in Scatter Transmission System, IEEE Trans. Communication Systems, 8, 56-57.

Gourieroux, C., Laurent, J. P., \& Scaillet O. (2000). Sensitivity Analysis of Values at Risk. Journal of Empirical Finance, 7, 225-245.

Heston, S. L., (1993). A Closed-Form Solution for Options with Stochastic Volatility with Applications to Bond and Currency Options. Review of Financial Studies, 6, 327-343.

Hull, J., \& White, A. (2012). CVA and Wrong Way Risk. Financial Analysis Journal, 68, 58-69. https://doi.org/10.2469/faj.v68.n5.6

Rosen, D., \& Saunders D. (2012). CVA the Wrong Way. Journal of Risk Management in Financial Institutions, 5, 252-272.

Slime, B. (2016). Credit Name Concentration Risk: Granularity Adjustment Approximation. Journal of Financial Risk Management, 5, 246-263.

https://doi.org/10.4236/jfrm.2016.54023

Scientific Research Publishing

Submit or recommend next manuscript to SCIRP and we will provide best service for you:

Accepting pre-submission inquiries through Email, Facebook, LinkedIn, Twitter, etc. A wide selection of journals (inclusive of 9 subjects, more than 200 journals)

Providing 24-hour high-quality service

User-friendly online submission system

Fair and swift peer-review system

Efficient typesetting and proofreading procedure

Display of the result of downloads and visits, as well as the number of cited articles

Maximum dissemination of your research work

Submit your manuscript at: http://papersubmission.scirp.org/

Or contact jfrm@scirp.org 